\title{
Effect of a Short-Term Online Version of a Mindfulness-Based Intervention on Self-criticism and Self-compassion in a Nonclinical Sample
}

\author{
Júlia Halamová \\ Institute of Applied Psychology, Faculty of Social \\ and Economic Sciences, Comenius University,
} Bratislava, Slovak Republic

Veronika Jurková

Institute of Applied Psychology, Faculty of Social and Economic Sciences, Comenius University, Bratislava, Slovak Republic

\author{
Martin Kanovský \\ Institute of Social Anthropology, Faculty of Social \\ and Economic Sciences, Comenius University, \\ Bratislava, Slovak Republic \\ Nuriye Kupeli \\ Marie Curie Palliative Care Research Department, \\ Division of Psychiatry, University College London, \\ United Kingdom
}

\begin{abstract}
Our goal was to investigate the efficacy of a Mindfulness-Based Intervention (MBI) in the form of a short-term, online intervention using exercises from Mindfulness-Based Stress-Reduction program on self-compassion, self-reassurance and self-criticism in a non-clinical population. We conducted pre-, post- and two-month follow-up measures of self-compassion, self-reassurance and self-criticism. A total of 146 participants, recruited through convenience sampling, were randomly allocated to the intervention with daily exercises for consecutive 15 days and to a control condition with no treatment. The intervention group reported a significant reduction in self-criticism and self-uncompassionate responding with effects present at two-month follow-up. There was a short-term effect of the training on self-compassion with no effect present at the two-month follow-up and no significant effect on self-reassurance. A limitation of the study is that participants' previous experience with meditation was not assessed, and thus the findings may be a result of previous meditation practice and not the intervention itself. Despite this limitation, the findings show that an online short-term MBI may be helpful in reducing selfcriticism in general population, but a larger study taking into account the limitations needs to be conducted to replicate this effect before recommendations for clinical practice can be made.
\end{abstract}

Key words: self-compassion, self-criticism, self-reassurance, mindfulness-based intervention, experiment

\section{Introduction}

Mindfulness is non-judgmental and accepting awareness of moment-to-moment experi- ence (Kabat-Zinn, 1990). Over the last two decades, mindfulness has become a popular target for interventions with clinical and nonclinical populations (Spijkerman, Pots, \& Bohlmeijer, 2016). Importantly, easy-to-administer methods

\footnotetext{
Acknowledgement: We would like to acknowledge Kristína Jakubcová, Alexandra Pačutová and Karolína Varšová for help with data collection and reaching consensus about the selection of the MBSR exercises implemented in the shortened version of the intervention. This work was supported by the Vedecká grantová agentúra VEGA (Grant number: 1/0578/15). Nuriye Kupeli was supported by Marie Curie core grant funding, grant MCCC-FCO-16-U.

Correspondence concerning this article should be addressed to Júlia Halamová, Institute of Applied Psychology, Faculty of Social and Economic Sciences, Comenius University in Bratislava, Mlynské luhy 4, 82105 Bratislava, Slovak Republic. E-mail: julia.halamova@gmail.com
}

Received May 19, 2018 
such as web-based mindfulness interventions are suggested to be effective in reducing stress and increasing mindfulness and well-being (Spijkerman et al., 2016). By the means of this non-judgmental and accepting awareness of momentary experience, mindfulness interventions are expected to alleviate unpleasant emotions (Keng, Smoski, \& Robins, 2011). Several approaches to teach ways to cultivate mindfulness already exist. According to Neff and Germer (2013), the most commonly offered Mindfulness-Based Intervention (MBI) is the Mindfulness-Based Stress Reduction (MBSR; KabatZinn, 1990). In addition to MBSR, Spijkerman et al. (2016) also refer to Mindfulness-Based Cognitive Therapy (MBCT; Teasdale, Segal, \& Williams, 1995), Dialectical Behaviour Therapy (DBT; Linehan, 1993), and Acceptance and Commitment Therapy (ACT; Hayes, Strosahl, \& Wilson, 1999) as the most frequently studied MBI.

\section{Mindfulness-Based Stress Reduction Program}

The MBSR program (Kabat-Zinn, 1990) is designed to support ways to cultivate mindfulness. Cultivating mindfulness improves the ability to be adaptive and deal effectively with stressful mental and physical experiences (Kabat-Zinn, 1990). MBSR is provided in groups by certified teachers. MBSR generally consists of a body scan exercise with awareness of body, yoga with awareness of movement or postures, sitting meditation with awareness of breathing and loving kindness meditation with awareness of human kind, which are all considered to be formal mindfulness practices and an informal mindfulness practice with awareness of activities in everyday life (MBSR Training Online, 2017) like washing dishes, driving or taking a shower. The manualized structured group sessions include training in formal and informal mindfulness practices as well as group interac- tion about their experience using mindfulness in their everyday lives. Informal practices aim to support the use of mindfulness in everyday life by being aware of one's own experience from moment to moment, while formal practices require deliberate commitment to practice mindfulness through guided awareness.

\section{The Impact of MBSR}

To date, several studies and meta-analyses have shown the MBSR program to be effective in reducing physical and mental symptoms and improving quality of life for people with a variety of physical and mental problems as well as for healthy individuals (e.g., Chiesa \& Serretti, 2009; Grossman et al., 2004; Huang et al., 2015; Khoury et al., 2015). Specifically, the MBSR is suggested to reduce stress, anxiety, depression, burnout and increase quality of life in nonclinical samples (Chiesa \& Serretti, 2009; Khoury et al., 2015). In summary, these meta-analyses suggest that MBSR is effective in reducing stress and unpleasant emotions related to negative experiences in both clinical and nonclinical samples but that there are varying degrees of effect across these outcomes. Moreover, there are some critical studies on MBSR (e.g., Dobkin, Irving, \& Amar, 2012) suggesting increases in intense reactions, stress or depression for some of the participants because of being more aware of unpleasant emotions, thoughts, or interpersonal problems. Also, Farias and Wikholm (2016) proposed that the effects of MBSR are only sustainable over short periods of time.

\section{The Impact of MBSR on Self-Compassion}

A resent meta-analysis of Zessin, Dickhâuser and Garbade (2015) demonstrated that there is a strong positive relationship between selfcompassion and well-being. Although MBSR 
(Kabat-Zinn, 1982) was originally developed to alleviate suffering from stress, it also promotes the cultivation of self-compassion with several studies demonstrating that MBSR is related to increases in self-compassion (e.g., Birnie et al., 2010; Robins et al., 2012; Shapiro et al., 2005; Shapiro et al., 2007). However, other studies have not always found a positive effect of MBSR on self-compassion (Abercrombie et al., 2007; Jazaieri et al., 2012; Shapiro et al., 2011). According to Keng, Smoski, Robins, Ekblad, and Brantley (2012), self-compassion might be a key process of change responsible for MBSR effectiveness. Therefore, in this study, we are interested in investigating the effect of an online MBI based on MBSR on self-compassion. To date, many studies have documented the positive effect of interventions designed to cultivate self-compassion (e.g., Kirby, 2017) but there are so far inconsistent findings about the effect of MBSR on self-compassion. In addition, no study to date has explored the effect of MBSR on self-criticism, which has been suggested to decrease through cultivating self-compassion (Gilbert, 2010). Self-criticism has been shown to be related to stress (e.g., Priel \& Shahar, 2000) and to be a major underlying factor for psychopathology (e.g., Falconer, King, \& Brewin, 2015). As a result, MBSR programs have been designed to target the effects of stress (e.g., Priel \& Shahar, 2000). People who indicate high levels of self-criticism have also been found to be reactive and sensitive to stressors (Dunkley, Zuroff, \& Blankstein, 2003). We hypothesize that if MBSR affects stress levels (Khoury et al., 2015), then MBSR and interventions based on MBSR should produce an effect on self-criticism. However, previous studies suggest that self-compassion and self-criticism are not simply the opposite poles of the same construct, and that the relationship between these constructs remains unclear (Gilbert, McEwan, Matos, \& Rivis, 2011; Neff, 2003). However, a caveat of these findings is that all the mentioned studies used the total score of the Self-Compassion Scale (SCS; Neff, 2003) to assess levels of self-compassion. Recent research on the factor structure of the SCS suggested that using the total score is not recommended and that positive and negative items should be calculated separately (e.g., López et al., 2015). This may explain the inconsistent findings between the studies. These conflicting findings suggest that the possible influence of the MBSR on selfcompassion needs further testing. Finally, although increasing self-compassion should influence a decrease in self-criticism (Gilbert et al., 2004), to our knowledge, no study to date has explored the impact of MBSR on self-criticism. Thus, it is necessary to explore the possible impact of the MBI based on MBSR on self-compassion and self-criticism.

\section{Adaptations to MBSR}

The MBSR program was developed as an eight-week course, with participants completing a weekly 2.5-hour session for eight consecutive weeks and a one-day six-hour session. According to previous research (Carmody, 2008), 45\% participants declined to participate in the study because of the time commitment required to complete the program. In addition, Carmody and Baer (2009) showed that there was no evidence that the shortened versions of MBSR were less effective than the standard format in reducing psychological distress. In support of this, several studies have reported a positive effect of MBSR on various outcomes such as depression, anxiety, mindfulness, positive moods, burnout symptoms, relaxation, and life satisfaction, when delivered as a shortened version (Abercrombie et al., 2007; Hallman et al., 2014; Jain et al., 2007; Speca et al., 2000; Klatt et al., 2009; Mackenzie et al., 2006). These findings illustrate that the benefits of MBSR can be achieved without committing too much time to completing the program. 


\section{Online Implementations of MBSR}

Delivering interventions using an online format can be cost-effective and convenient due to improved access, quicker provision of care because of no waiting list and no travelling time to sessions, less or no direct health professional input reducing the stigma of being labelled as a patient or client with a mental health condition, and automatic monitoring of progress through online assessments (Andersson \& Titov, 2014; Cuijpers et al., 2009).

Although the majority of MBSR sessions are delivered in group format (e.g., Kabat-Zinn, 1982; Kabat-Zinn, 2013; Kabat-Zinn, 2016), 42\% of people would prefer to complete mindfulness meditation on their own using an online format, compared to completing it during group or individual face-to-face sessions (Wahbeh et al., 2014). Despite the popularity of online-based interventions, there are a limited number of RCTs examining the effectiveness of online based MBSR programs (Aikens et al., 2014; Glück \& Maercker, 2011; Mak, Chan, Cheung, Lin, \& Ngai, 2015; Morledge et al., 2013; Wolever et al., 2012; Zernicke et al., 2014). Participants, who completed the online-based MBSR program from Aikens et al. (2014), reported significant decreases in perceived stress as well as an increase in mindfulness, resiliency, and vigour. Similarly, the study by Mak et al. (2015) displayed significant improvements in mindfulness as well as mental well-being. In the study of Morledge et al. (2013), the online adaptation of the MBSR significantly improved perceived stress, mindfulness, self-transcendence, psychological well-being, and quality of life. Research study of Wolever et al. (2012) showed significant improvements on perceived level of stress, quality of sleep, and heart rate variability. In the study of Zernicke et al. (2014), the online mindfulness-based intervention significantly improved mood disturbance, stress symptoms, spirituality, mindful behavior, posttraumatic growth, and mindfulness. All of the changes were compared with the control groups in all the mentioned online research studies. In addition, Wolever et al. (2012) tested difference in effectiveness of either group or online versions of the mindfulness program with basically equivalent results of those two types of program delivery. Contrary, Glück and Maercker (2011) did not find any significant effects of their mindful intervention. However, analysis of persons with over $50 \%$ participation discovered significant effects for perceived stress and negative emotions.

Although there are several studies on the effectiveness of MBSR and its adaptations, to our knowledge, no study to date has examined the impact of the MBI based on MBSR on selfcompassion and self-criticism concurrently.

\section{Aims}

The primary aim of the current study was to evaluate the immediate and longer-term impact of a 15-day internet-based MBI consisting of MBSR exercises on self-compassion, self-criticism and self-reassurance in a non-clinical population.

\section{Methods}

\section{Trial Design}

We used open parallel-groups design of RCT. All participants completed demographic information and baseline measures and were then allocated using block randomization to the intervention and control groups knowing to which group theybelong. To enable random allocation into the two conditions, the first fifteen participants were allocated to the intervention condition and the nexteight participants were allocated to the control condition. This was done until all participants were allocated into the two condi- 
tions. More participants were allocated to the intervention group because due to the time commitment required of the intervention group, we expected higher attrition for the intervention group. The control group was not provided with any further instructions until 15 days after completing the baseline measures when they received an email to a link to complete the online self-report measures. This process was repeated for the two-month follow-up.

\section{Participants}

Participants were recruited from the general community through social media, social networking sites and health and well-being forums in Slovakia. As a gesture of gratitude, those who completed the study were entered into a prize draw to win a tablet. The only inclusion criterion was adults over the age of 18 years.

A total of 146 participants completed the preintervention measures and from this sample, 93 were randomly allocated to the intervention group and 53 were assigned to the control condition. From this sample, 42 participants from the intervention group and 23 participants from the control group completed the post-intervention measures. All 42 participants completing post-intervention measures in the MBI group completed the two-month follow-up and 20 of the 23 participants of the control group completed the follow-up measures (see Figure 1 for

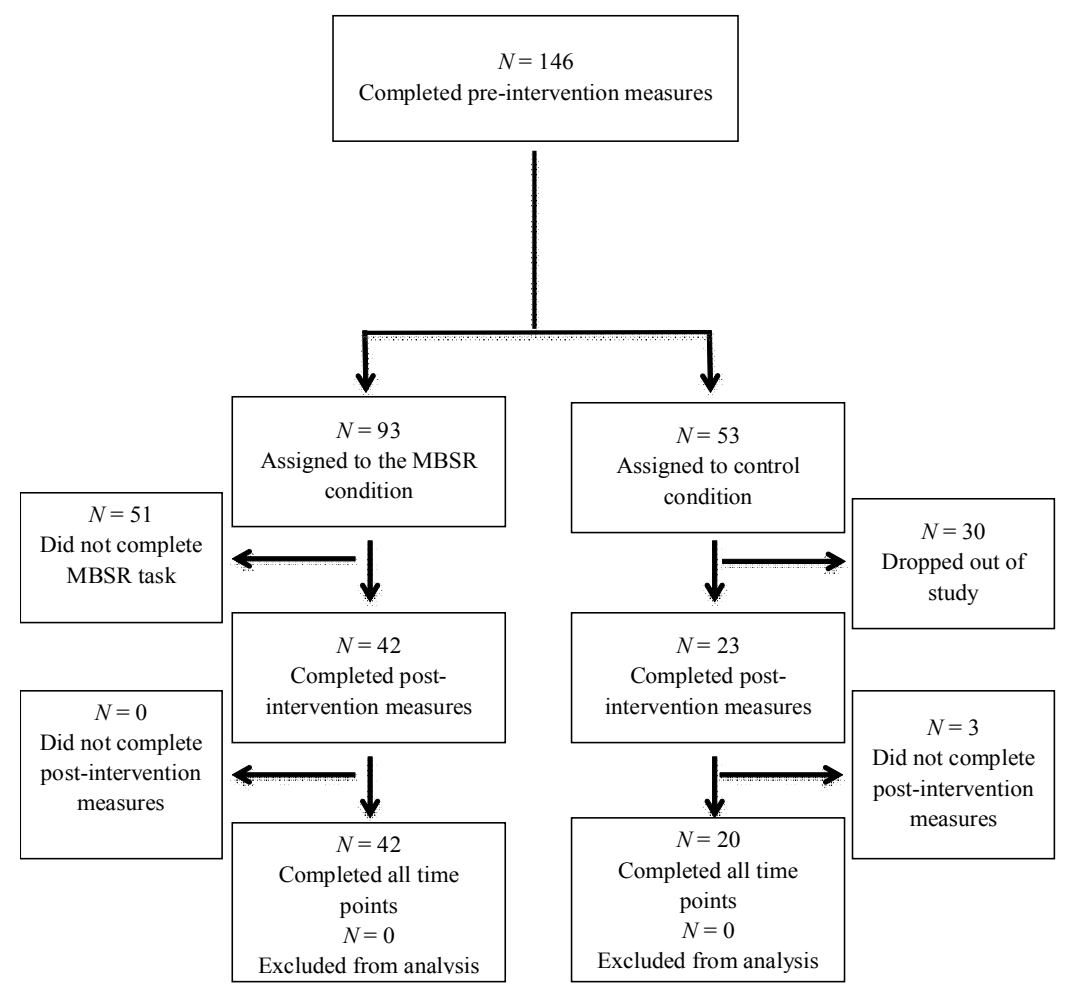

Figure 1 Flow chart for the number of participants who completed each phase of the study and attrition 
study attrition). The final control group consisted of 17 women and 3 men with a mean age of 25.35 years $(S D=6.32)$ and the intervention group consisted of 36 women and 6 men with mean age of 25.57 years $(S D=11.76)$. Results show that no significant differences were present between those who completed the intervention and those who dropped out, for all SCS subscales and for all FSCRS subscales ( $p$-values 0.170$)$.

\section{Intervention}

Participants assigned to the MBI condition were instructed to complete a daily MBSR exercise for 15 consecutive days and were advised to commit 15 minutes each day to practice each exercise. To make it comparable to other studies, a number of intervention days was determined in order to increase accessibility of the intervention for the participants, along with achieving at least some effectiveness, based on previous research studies with two-weeks interventions (e.g., Banerjee, Cavanagh, \& Strauss, 2018). Participant's assigned to the intervention group received an email prompting them to complete the MBSR task and each participant received the same exercise each day in the same order (i.e., the order of the exercises was not randomized). All emails were presented in the same format consisting of a short introduction in the form of psychoeducation, explaining the intended impact of the exercise in order to motivate participants to complete it, instructions for the exercise, and post-exercise questions. These were designed to encourage participants to reflect on the experience in order to increase and imbed the impact of the exercise. The additional function of the post-intervention questions following each exercise was to check if participants performed the exercise. If the participant had not completed the exercise, they were sent an email reminder.
The tasks were selected by consensus of our research team from different exercises available from previous publications on MBSR (e.g., Kabat-Zinn, 1982; Kabat-Zinn, 2013; KabatZinn, 2016; MBSR Palouse Mindfulness, 2017; MBSR Training Online, 2017) and approved by a certified trainer of MBSR in Slovakia. Majority of the exercises were presented in the form of an audio recording, the yoga exercise was presented in the form of pictures. All the exercises as well as audio recordings were translated into Slovak. The intervention was accessible on any computer or smartphone via a link on the day the email was sent. For the first three days, participants practiced "Body Scan", followed by "Sitting meditation", then "Yoga", and the last exercises were "Loving Kindness Meditation", and "Informal mindfulness practices". Following the final exercise, participants were instructed to complete the post-intervention measures and this was repeated at the twomonth follow-up.

\section{Measures}

Self-criticism/reassurance was assessed using the Forms of Self-Criticising/Attacking \& Self-Reassuring Scale (FSCRS; Gilbert et al., 2004). The FSCRS is a 22-item measure requiring participants to rate statements on a 5-point Likert scale. Positive items reflect the ability to self-reassure (referred to as Reassured self) and negative items indicate self-critical thoughts and feelings (split into subscales of Inadequate self and Hated self). This scale has been validated in various samples in different countries (e.g., Castilho, Pinto-Gouveia, \& Duarte, 2015; Kupeli, Chilcot, Schmidt, Campbell, \& Troop, 2013) including Slovakia (Halamová, Kanovský, \& Pacúchová, 2017a). According to these studies, FSCRS has good psychometric properties including reliability and validity. In this study, Cronbach's alpha for Inadequate self was 0.87 , for Hated self 0.68 , and for Reassured self 0.86 . 
Self-compassion was assessed using the SelfCompassion Scale (SCS; Neff, 2003). The SCS measures six components of self-compassion experienced during perceived difficulty. The scale consists of 26 items rated on a 5-point Likert-type scale. The scale consists of six subscales that measure the degree to which individuals display self-kindness against selfjudgment, common humanity versus isolation, and mindfulness versus over-identification. Recent findings demonstrated that the negative and positive subscales of the SCS should be calculated separately and not summed as a single score (e.g., Brenner, Heath, Vogel, \& Credé, 2017; López et al., 2015; Muris \& Petrocchi, 2017). According to these studies, SCS has good reliability and validity. These findings have been replicated using the Slovak version of the scale (Halamová, Kanovský, \& Pacúchová, 2017b). Therefore, for the purpose of this study, the combined score of the positive constructs will be used as a reflection of self-compassionate responding (self-kindness, common humanity and mindfulness) and the combined score of the negative constructs will reflect self-uncompassionate responding (selfjudgement, isolation and over-identification). In this study, Cronbach's alpha for Self-compassionate responding was 0.88 , and for Selfuncompassionate responding was 0.89 .

\section{Data Analyses}

For the statistical analysis, program R (Version 3. 4. 0, R Core Team, 2017), and the package nparLD (Noguchi et al., 2012) were used.

Factorial designs are usually analyzed by means of parametric procedures (ANOVA). However, the assumptions of parametric methods such as homoscedasticity, normality, or absence of outliers are seldom met in practice. Classical non-parametric alternatives (WilcoxonMann-Whitney test, Kruskal-Wallis test, Waldtype statistics) perform poorly for small sample sizes, heteroscedasticity and unbalanced designs (when the size of control and the experimental sample are different; see Brunner et al., 1999; Brunner et al., 2002; Brunner et al., 2016). Our dependent variables are raw scores of ordinal items, so we cannot just assume their normal distribution, and intervention design practically excludes equal variances of control and experimental groups (see Tables 2 and 4). Therefore, our data are heteroscedastic, as it should be: intervention usually decreases variance in its group. We will report ANOVA-type statistics (Brunner et al., 2016) from non-parametric rank-based test for longitudinal data, and relative effects, which can serve as effect size measures. The relative effect can be regarded as the probability that a randomly chosen observation from the treatment group takes on larger values than an observation randomly chosen from the mean distribution function. Therefore, a relative effect significantly higher (for increasing effect) or lower (for decreasing effect) than 0.50 indicates that an intervention was effective. ANOVA-type statistics (ATS) performs well even for small sample sizes and unbalanced designs (Brunner et al., 2002).

In this design, it is mainly of interest to investigate an interaction between groups (factor $\mathrm{G}$ ) and time (factor $\mathrm{T}$ ). There is a control group without intervention (group 1) and the active treatment is given to an intervention group (group 2), therefore, the distribution functions at the start of the trial (time point 1) are identical because the subjects were randomly assigned to the two groups of factor G. Then, an effect of the active intervention should produce nonparallel time curves of the measurements. This means that there should be a significant interaction between factor $\mathrm{G}$ and factor $\mathrm{T}$ if the intervention is effective. We hypothesize that our intervention will be significantly effective if and only if the interaction between group (control vs. intervention) and time (three time points) is significant: the significant difference between 
control and experimental group and/or between time points alone will not do. Main factorial effects (difference between groups regardless of time, or difference among time points regardless of groups) are of no use here, so we will not report them.

Data analyses were decided before the data were gathered and it was selected because this statistical method was recently developed for non-parametric analysis of longitudinal data in parallel and factorial experiments. These nonparametric methods allow for robust statistical analysis of small sample sizes and unbalanced designs (Delaney \& Vargha, 2002; Erceg-Hurn $\&$ Mirosevich, 2008).

\section{Results}

Before testing our hypothesis, we conducted preliminary analyses to ensure participants were successfully randomized into the two groups. Since distributions and variances of groups are almost equal, we used the nonparametric Wilcoxon-Mann-Whitney test to compare baseline scores for the intervention and control groups. Results show that no significant differences occurred ( $p$-values range from 0.22 to 0.83 for all SCS subscales, and from 0.15 to 0.71 for all FSCRS subscales).

The results revealed that there was a significant immediate effect of the intervention on selfcriticism (Inadequate self and Hated self) and
Inadequate self and longitudinal change on Hated self (see Table 1 and Table 2). There was no effect of the intervention on Reassured self. Relative effects with their confidence intervals for each group and time point illustrate that the effect of the intervention on self-criticism and its subscales is present at the two-month follow-up. A comparison of the relative effects for Hated self and Reassured self shows that there is a significant and persistent change in Hated self but no effect on Reassured self.

There was an effect of the intervention on the total score of the SCS (see Table 3), but this effect is due to a reduction in Self-uncompassionate responding (negative items). The effect of the intervention on self-uncompassionate responding scores significantly decreased immediately post-intervention and at the twomonth follow-up. There was an immediate effect of the intervention on the Self-compassionate responding subscale but no effect of intervention at the two-month follow-up. Again, relative effects with their confidence intervals for each group and time point is presented in Table 4 , which illustrates these effects in clearer detail.

Relative marginal effects express the strength of the effect based on the magnitude of their distance from .50 (null hypothesis: the probability is at random). Based on this criterion, effect sizes are small $(0.50-0.60$ or $0.40-$ $0.50)$.

Table 1 Results for interaction effects of the FSCRS scale

\begin{tabular}{llll}
\hline & \multicolumn{3}{c}{$A T S$} \\
\cline { 2 - 4 } & $F$ & $d f$ & $p$ \\
\hline FSCRS Reassured self & 0.03 & $1.54, \infty$ & 0.950 \\
FSCRS Inadequate + Hated self & 4.39 & $1.88, \infty$ & $0.014^{*}$ \\
FSCRS Inadequate self & 4.34 & $1.73, \infty$ & $0.017^{*}$ \\
FSCRS Hated self & 4.29 & $1.99, \infty$ & $0.014^{*}$ \\
\hline
\end{tabular}

Note. FSCRS - The Forms of Self-Criticising/Attacking \& Self-Reassuring Scale, ATS ANOVA Type Statistics, $F-$ F-ratio, $d f$ - degrees of freedom.

$* p<0.05, * * p<0.01, * * * p<0.001$. 
Table 2 Relative effects, their confidence intervals and variances of the FSCRS scale

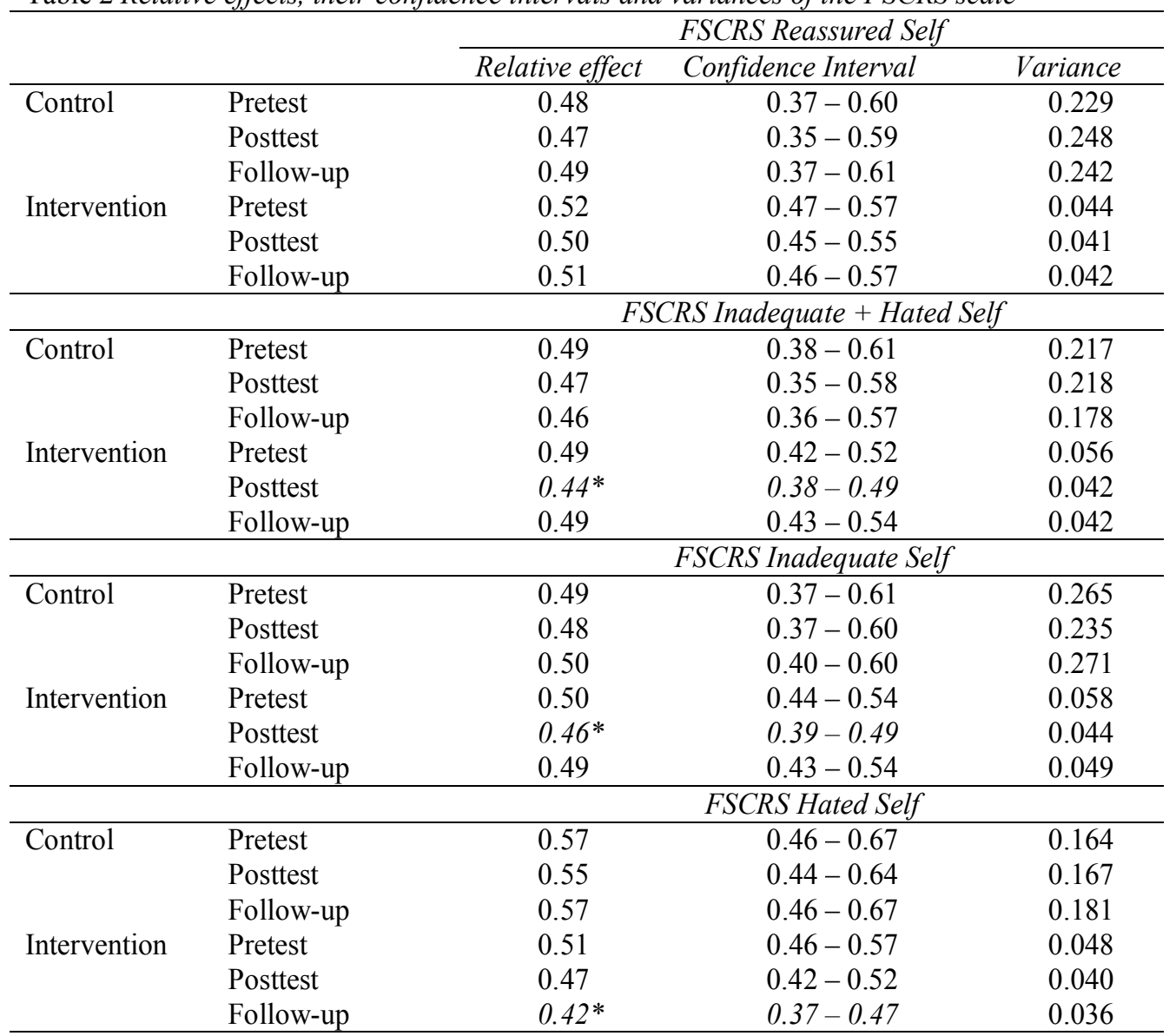

Note. FSCRS - The Forms of Self-Criticising/Attacking \& Self-Reassuring Scale.

$* p<0.05, * * p<0.01, * * * p<0.001$

Table 3 Results for interaction effects of the SCS scale

\begin{tabular}{llll}
\hline & \multicolumn{3}{c}{$A T S$} \\
\cline { 2 - 4 } & $F$ & $d f$ & $p$ \\
\hline SCS sum score & 8.11 & $1.86, \infty$ & $0.001^{*}$ \\
SCS positive (Self-compassionate responding) & 1.56 & $1.73, \infty$ & 0.213 \\
SCS negative (Self-uncompassionate responding) & 13.48 & $1.99, \infty$ & $0.001^{*}$ \\
\hline
\end{tabular}

Note. SCS - The Self-Compassion Scale, ATS - Anova Type Statistics, $F-$ F-ratio, $d f-$ degrees of freedom.

$* p<0.05, * * p<0.01, * * * p<0.001$ 
Table 4 Relative effects, their confidence intervals and variances of the SCS scale

\begin{tabular}{|c|c|c|c|c|}
\hline & & & SCS sum score & \\
\hline Group & Time point & Relative effect & Confidence Interval & Variance \\
\hline \multirow[t]{3}{*}{ Control } & Pretest & 0.47 & $0.35-0.60$ & 0.260 \\
\hline & Posttest & 0.49 & $0.38-0.62$ & 0.241 \\
\hline & Follow-up & 0.44 & $0.33-0.56$ & 0.219 \\
\hline \multirow[t]{4}{*}{ Intervention } & Pretest & 0.45 & $0.39-0.51$ & 0.055 \\
\hline & Posttest & $0.56^{*}$ & $0.51-0.62$ & 0.051 \\
\hline & Follow-up & $0.56^{*}$ & $0.51-0.62$ & 0.052 \\
\hline & & \multicolumn{3}{|c|}{ SCS positive (Self-compassionate responding) } \\
\hline \multirow[t]{3}{*}{ Control } & Pretest & 0.45 & $0.35-0.56$ & 0.202 \\
\hline & Posttest & 0.48 & $0.37-0.59$ & 0.204 \\
\hline & Follow-up & 0.47 & $0.35-0.59$ & 0.231 \\
\hline \multirow[t]{4}{*}{ Intervention } & Pretest & 0.47 & $0.42-0.52$ & 0.040 \\
\hline & Posttest & $0.59 *$ & $0.54-0.63$ & 0.033 \\
\hline & Follow-up & 0.49 & $0.45-0.54$ & 0.035 \\
\hline & & \multicolumn{3}{|c|}{ SCS negative (Self-uncompassionate responding) } \\
\hline \multirow[t]{3}{*}{ Control } & Pretest & 0.44 & $0.34-0.54$ & 0.149 \\
\hline & Posttest & 0.46 & $0.35-0.57$ & 0.144 \\
\hline & Follow-up & 0.42 & $0.32-0.53$ & 0.121 \\
\hline \multirow[t]{3}{*}{ Intervention } & Pretest & 0.43 & $0.33-0.53$ & 0.076 \\
\hline & Posttest & $0.60^{*}$ & $0.55-0.65$ & 0.060 \\
\hline & Follow-up & $0.58 *$ & $0.53-0.63$ & 0.061 \\
\hline
\end{tabular}

Note. SCS - The Self-Compassion Scale.

$* p<0.05, * * p<0.01, * * * p<0.001$.

\section{Discussion}

The present study examined the immediate and long-term effects of a 15-day internet-based MBI using exercises from the MindfulnessBased Stress Reduction (Kabat-Zinn, 1990) program on self-compassion and self-criticism. To our knowledge, this is the first study to examine the impact of MBI on self-criticism in a Slovak sample.

Cultivating mindfulness by means of MBI for 15 days decreased self-criticism, as measured by the FSCRS (Gilbert et al., 2004) and self-uncompassionate responding as measured by the SCS (Neff, 2003), immediately and these changes persisted over two months. In con- trast, practicing mindfulness was effective in increasing self-compassionate responding in the short term but this effect was not present at the two-month follow-up. There was no change in self-reassurance after the intervention, which could have been caused by possible difference between self-compassion and self-reassurance, even though Kupeli et al. (2013) suggest that these two constructs are the same.

We also explored the effect of mindfulness training on the dimensions of the scales separately. Our findings suggest that the MBI does not induce a persistent change in self-compassion, thus supporting previous research (Abercrombie et al., 2007; Jazaieri et al., 2012; Shapiro et al., 2011). Our findings did reveal a 
significant decrease in self-criticism (specifically Hated self and Inadequate self) and also in Selfuncompassionate responding. This is not surprising, as there is some similarity between the items for the FSCRS self-criticism and SCS critical self-judgement.

As MBSR was originally developed to alleviate suffering during adversity, it is quite plausible that it reduces the effects of stress resulting from self-critical thoughts and feelings. The generalized effect of MBI based on MBSR on stress targets self-criticism, which is represented by Hated self and Inadequate self, but the MBI had a different effect on these two forms of selfcriticism. MBI had a more immediate effect on IS and a slower effect on HS, which was present by Hated self being significant only in followup measures. The possible explanation of such significant effect for Hated self could be that subjects with higher levels of Hated self had higher inertia for any change and the effect was therefore delayed. Also, our differing findings related to Inadequate self and Hated self supported the findings of previous research study (Longe et al., 2010), which suggested that the experience of IS and HS activate different parts of the brain. Further research is required to address this issue.

It is true that reported effect sizes were small when considered separately. However, they showed a systematic pattern: the main impact was on negative parts (Hated self, Inadequate self, Self-Uncompassionate Responding), which refers to systematic effectivity of intervention despite its small effect sizes.

The present findings also support the idea that the total score of the SCS (Neff, 2003) may not be useful, either for research or for practice (e.g., López et al., 2015). In our study, the total score of the SCS showed significant increase but this effect was due to a decrease in selfuncompassionate responding since the effect on self-compassionate responding was short lived.

\section{Limitations}

The current study did not involve certified MBSR teachers in implementing the intervention, which is the major limitation of the study. However, we selected exercises from the original MBSR to emulate sufficient exposure to mindfulness, and the selection was approved by a certified trainer of MBSR in Slovakia. Also, as this study recruited a sample from the general population, these findings cannot be generalized to the clinical population and thus, this adaptation to the MBI needs to be evaluated with people with psychological morbidity. Another limitation of the present study is that a smaller proportion of men took part in the study and thus these findings may not be applicable to men. However, previous research has suggested that gender differences are not evident when assessing the effectiveness of MBSR (Shapiro et al., 2007). Nonetheless, further research on how men and women respond to MBSR, MBI or their online version is required.

Also, the sample in the study was not representative of the general population, since there were mostly young adults. Therefore, the findings in our study have only limited generalizability and practical implications.

Another limitation of the study is that participants were not initially assessed in terms of their familiarity with mindfulness and related practices, so we do not know how many of them had previous experience with mindfulness.

As we did not include a measure of mindfulness, it is difficult to quantify the overall efficacy of the shortened program and compare it with studies investigating other versions of the program.

Moreover, we did not measure participants' previous experience with meditation. So, we are not able to eliminate the possibility that our significant findings are a result of previous medi- 
tation practice and not the online intervention itself.

In addition, there is a limitation of a non-treatment control condition used in this study. All effects could possibly be attributed to the demand effects because receiving some kind of treatment might encourage participants in the intervention group to indicate that there was some improvement, simply because they believe that this is what they are supposed to say.

As the research relied on self-report measures of self-compassion and self-criticism, it could potentially be biased for socially desirable responding, however, these measures have been shown to have good psychometric properties (Halamová, Kanovský, \& Pacúchová, 2017a, 2017b). Therefore, future research should assess outcomes using objective measures such as physiological measures (e.g., heart rate variability, respiration rate variability).

Furthermore, Cronbach's alpha for Hated self (FSCRS) was .68, which is slightly below the recommended guidelines (Lance, Butts, \& Michels, 2006) and might be attributed to the small size of our sample. However, this subscale showed acceptable psychometric properties in previous research in Slovak population (Halamová, Kanovský, \& Pacúchová, 2017a).

\section{Conclusion}

An abbreviated and web-based version of the Mindfulness-Based Intervention has significantly decreased self-criticism and selfuncompassionate responding with effects lasting at least two months. It also increased selfcompassionate responding, but these effects wereshort-lived. These results are promising and posit that interventions can be provided using cost-effective methods and be accessible for broader populations without direct involvement of mental health professionals. This is particularly relevant to those who might be unable or reluctant tocontact a mental health care provider.

\section{References}

Aikens, K. A., Astin, J., Pelletier, K. R., Levanovich, K., Baase, C. M., Park, Y. Y., \& Bodnar, C. M. (2014). Mindfulness goes to work: Impact of an online workplace intervention. Journal of Occupational and Environmental Medicine, 56(7), 721731. doi: 10.1097/JOM.0000000000000209

Andersson, G., \& Titov, N. (2014). Advantages and limitations of Internet-based interventions for common mental disorders. World Psychiatry, 13(1), 411. doi: 10.1002/wps.20083.

Abercrombie, P. D., Zamora, A., \& Korn, A. P. (2007). Lessons learned: Providing a mindfulness-based stress reduction program for low-income multiethnic women with abnormal pap smears. Holistic Nursing Practice, 21(1), 26-34.

Banerjee, M., Cavanagh, K., \& Strauss, C. (2018). Barriers to mindfulness: A path analytic model exploring the role of rumination and worry in predicting psychological and physical engagement in an online mindfulness-based intervention. Mindfulness, 9(3), 980-992. doi:10.1007/s12671-017-0837-4

Birnie, K., Speca, M., \& Carlson, L. E. (2010). Exploring self-compassion and empathy in the context of Mindfulness-Based Stress Reduction (MBSR). Stress and Health, 26, 359-371. doi:10.1002/ smi.1305

Brenner, R. E., Heath, P. J., Vogel, D. L., \& Credé, M. (2017). Two is more valid than one: Examining the factor structure of the Self-Compassion Scale (SCS). Journal of Counseling Psychology, 64(6), 696-707. doi: $10.1037 /$ cou0000211

Brunner, E., Munzel, U., \& Puri, M. L. (1999). RankScore Tests in factorial designs with repeated measures. Journal of Multivariate Analysis, 70, 286317. doi: 10.1006/jmva.1999.1821

Brunner, E., Domhof, S., \& Langer, F. (2002). Nonparametric analysis of longitudinal data in factorial designs. Wiley, New York.

Brunner, E., Konietschke, F., Pauly, M., \& Puri, M. L. (2016). Rank-based procedures in factorial designs: Hypotheses about non-parametric treatment effects. Journal of the Royal Statistical Society, Series B. doi:10.1111/rssb.12222.

Castilho, P., Pinto-Gouveia, J., \& Duarte J. (2015). Exploring self-criticism: Confirmatory factor analysis of the FSCRS in clinical and nonclinical samples. Clinical Psychology \& Psychotherapy, 22(2), 153164.

Carmody, J. (2008). Mindfulness-based stress reduction for asthma. Unpublished data, University of Massachusetts Medical School, Worcester, MA. 
Carmody, J., \& Baer, R. A. (2009). How long does a mindfulness-based stress reduction program need to be? A review of class contact hours and effect sizes for psychological distress. Journal of Clinical Psychology. 65(6), 627-638. doi: 10.1002/ jclp. 20555

Chiesa, A., \& Serretti, A. (2009). Mindfulness-based stress reduction for stress management in healthy people: A review and meta-analysis. The Journal of Alternative and Complementary Medicine, 15, 593600. doi:10.1089/acm.2008.0495

Cuijpers, P., Marks, I. M., van Straten, A., Cavanagh, K., Gega, L., \& Andersson, G. (2009). Computeraided psychotherapy for anxiety disorders: A metaanalytic review. Cognitive Behavioral Therapy, 38(2), 66-82. doi: 10.1080/16506070802694776

Delaney, H. D., \& Vargha, A. (2002). Comparing several robust tests of stochastic equality with ordinally scaled variables and small to moderate sized samples. Psychological Methods, 7, 485-503.

Dobkin, P., Irving, J., \& Amar, S. (2012). For whom may participation in a mindfulness-based stress reduction program be contraindicated? Mindfulness, 3(1), 44-50. doi:10.1007/s12671-011-0079-9

Dunkley, D. M., Zuroff, D. C., \& Blankstein, K. R. (2003). Self-critical perfectionism and daily affect: Dispositional and situational influences on stress and coping. Journal of Personality and Social Psychology, 84, 234-252. doi: 10.1037/0022-3514.84. 1.234

Erceg-Hurn, D., \& Mirosevich, V. M. (2008). Modern robust statistical methods: An easy way to maximize the accuracy and power of your research. American Psychologist, 63(7), 591-601. doi: 10.1037/0003066X.63.7.591.

Falconer, C. J., King, J. A., \& Brewin, C. R. (2015). Demonstrating mood repair with a situation-based measure of self-compassion and self-criticism. Psychology and Psychotherapy, 88(4), 351-365. doi: 10.1111/papt.12056

Farias, M., \& Wikholm, C. (2016). Has the science of mindfulness lost its mind? BJPsych Bulletin, 40(6), 329-332.doi: 10.1192/pb.bp.116.053686.

Gilbert, P. (2010). Training our minds in, with and for compassion. An introduction to concepts and compassion-focused exercises. Retrieved on 21.8.2016 from: http://wtm.thebreathproject.org/ wp-content/uploads/2016/03/COMPASSIONHANDOUT.pdf

Gilbert, P., Clark, M., Hempel, S., Miles, J. N. V., \& Irons, C. (2004). Criticising and reassuring oneself: An exploration of forms, styles and reasons in female students. British Journal of Clinical Psychology, 43, 31-50. doi: 10.1348/ 014466504772812959
Gilbert, P., McEwan. K., Matos, M., \& Rivis, A. (2011) Fears of compassion: Development of three selfreport measures. Psychology and Psychotherapy: Theory, Research and Practice, 84, 239-255. doi: 10.1348/147608310X526511

Glück, T., \& Maercker, A. (2011). A randomized controlled pilot study of a brief web-based mindfulness training. BMC Psychiatry, 11, 175-179.

Grossman, P., Niemann, L., Schmidt, S., \& Walach, H. (2004). Mindfulness-based stress reduction and health benefits. A meta-analysis. Journal of Psychosomatic Research, 57, 35-43. doi:10.1016/S00223999(03)00573-7

Halamová, J., Kanovský, M., \& Pacúchová M. (2017a). Robust psychometric analysis and factor structure of the Forms of Self-criticizing/Attacking and Self-reassuring Scale. Československá Psychologie, 4, 456-471.

Halamová, J., Kanovský, M., \& Pacúchová M. (2017b). Self-Compassion Scale: IRT psychometric analysis validation and factor structure - Slovak translation. Psychologica Belgica, 57(4), 190-209. doi: $10.5334 / \mathrm{pb} .398$

Hallman, I. S., O'Connor, N., Hasenau, S., \& Brady, S. (2014). Improving the culture of safety on a highacuity inpatient child/adolescent psychiatric unit by mindfulness-based stress reduction training of staff. Journal of Child and Adolescent Psychiatric Nursing, 27(4), 183-189. doi:10.1111/jcap.12091

Hayes, S. C., Strosahl, K., \& Wilson, K. G. (1999). Acceptance and commitment therapy. New York: Guilford Press.

Huang, H. P., He, M., Wang, H. Y., \& Zhou, M. A. (2016). A meta-analysis of the benefits of mindfulness-based stress reduction (MBSR) on psychological function among breast cancer (BC) survivors. Breast Cancer, 23(4), 568-576. doi: 10.1007/ s12282-015-0604-0

Jain, S., Shapiro, S. L., Swanick, S., Roesch, S. C., Mills, P. J., Bell, I., \& Schwartz, G. E. R. (2007). A randomized controlled trial of mindfulness meditation versus relaxation training: Effects on distress, positive states of mind, rumination, and distraction. Annals of Behavioral Medicine, 33(1), 11-21. doi: 10.1207/s15324796abm3301_2

Jazaieri, H., Goldin, P. R., Werner, K., Ziv, M., \& Gross, J. J. (2012). A randomized trial of mindfulness-based stress reduction versus aerobic exercise for social anxiety disorder. Journal of Clinical Psychology, 68, 715-731. doi:10.1002/jclp.21863

Kabat-Zinn, J. (1982). An out-patient program in behavioral medicine for chronic pain patients based on the practice of mindfulness meditation: Theoretical considerations and preliminary results. General Hospital Psychiatry, 4, 33-47. 
Kabat-Zinn, J. (1990). Full catastrophe living: Using the wisdom of your body and mind to face stress, pain and illness. New York: Delacorte.

Kabat-Zinn, J. (2013). Guided mindfulness meditation. Series 1. New York: BetterListen! LLC.

Kabat-Zinn, J. (2016). Mindfulness for beginners: Reclaiming the present moment-and your life. Colorado: Sounds true.

Keng, S-L., Smoski, M. J., \& Robins, C. J. (2011). Effects of mindfulness on psychological health: A review of empirical studies. Clinical Psychology Review, 31, 1041-1056. doi: 10.1016/j.cpr.2011. 04.006

Keng, S., Smoski, M. J., Robins, C. J., Ekblad, A. G., \& Brantley, J. G. (2012). Mechanisms of change in mindfulness-based stress reduction: Self-compassion and mindfulness as mediators of intervention outcomes. Journal of Cognitive Psychotherapy, 26(3), 270-280. doi:10.1891/0889-8391.26.3.270

Kirby, J. N. (2017). Compassion interventions: The programs, the evidence, and implications for research and practice. Psychology and Psychotherapy: Theory, Research and Practice, 90(3), 432-455. doi: 10.1111/papt.12104

Khoury, B., Sharma, M., Rush, S. E., \& Fournier, C. (2015). Mindfulness-based stress reduction for healthy individuals: A meta-analysis. Journal of Psychosomatic Research, 78, 519-528. doi:10.1016/j. jpsychores.2015.03.009

Klatt, M. D., Buckworth, J., \& Malarkey, W. B. (2009). Effects of low-dose mindfulness-based stress reduction (MBSR-ld) on working adults. Health Education \& Behavior: The Official Publication of the Society for Public Health Education, 36(3), 601614. doi:10.1177/10901981083176

Kupeli, N., Chilcot, J., Schmidt, U. H., Campbell, I. C., $\&$ Troop, N. A. (2013). A confirmatory factor analysis and validation of the forms of Self-Criticism/ Reassurance Scale. British Journal of Clinical Psychology, 52(1), 12-25.

Lance, C. E., Butts, M. M., \& Michels, L. C. (2006). The sources of four commonly reported cutoff criteria: What did they really say? Organizational Research Methods, 9(2), 202-220. doi: 10.1177/ 1094428105284919

Linehan, M. M. (1993). Cognitive behavioral treatment of borderline personality disorder. New York: Guilford Press.

Longe, O., Maratos, F. A., Gilbert, P., Evans, G., Volker, F., Rockliff, H., \& Rippon, G. (2010). Having a word with yourself: Neural correlates of self-criticism and self-reassurance. Neuroimage, 49, 1849 1856. doi:10.1016/j.neuroimage.2009.09.019

López, A., Sanderman, R., Smink, A., Zhang, Y., van Sonderen, E., Ranchor, A., \& Schroevers, M. J.
(2015). A reconsideration of the Self-Compassion Scale's total score: Self-compassion versus self-criticism. PloS One, 10(7). doi: 10.1371/journal.pone. 0132940

Mak, W. W. S., Chan, A. T. Y., Cheung, E. Y. L., Lin, C. L. Y. \& Ngai, K. C. S. (2015). Enhancing Webbased mindfulness training for mental health promotion with the health action process approach: Randomized controlled trial. Journal of Medical Internet Research, 17(1), 8.

MBSR Palouse Mindfulness. (2017). Retrieved on 27.8.2016 from: https://palousemindfulness.com/ MBSR/manual.html

MBSR Training Online. (2017). Retrieved on 28.8.2016 from: http://www.mbsrtraining.com/

Mackenzie, C. S., Poulin, P. A., \& Seidman-Carlson, R. (2006). A brief mindfulness-based stress reduction intervention for nurses and nurse aides. Applied Nursing Research, 19, 105-109. doi: 10.1016/ j.apnr.2005.08.002

Morledge, T. J., Allexandre, D., Fox, E., Fu, A. Z., Higashi, M. K., Kruzikas, D. T.,... \& Reese, P. R. (2013). Feasibility of an online mindfulness program for stress management - A randomized, controlled trial. Annals of Behavioral Medicine, 46, 137-148. doi:10.1007/s12160-013-9490-x

Muris, P., \& Petrocchi, N. (2017). Protection or vulnerability? A meta-analysis of the relations between the positive and negative components of self-compassion and psychopathology. Clinical Psychology \& Psychotherapy, 24(2), 373-383. doi: 10.1002/ cpp. 2005

Neff, K. D. (2003). Development and validation of a scale to measure self-compassion. Self and Identity, 2, 223-250. doi: 10.1080/15298860309027

Neff, K. D., \& Germer, C. K. (2013). A pilot study and randomized controlled trial of the Mindful Self-Compassion Program. Journal of Clinical Psychology, 69(1), 28-44. doi: 10.1002/jclp.21923

Noguchi, K., Gel, Y., Brunner, E. \& Konietsche, F. (2012). nparLD: An R software package for the nonparametric analysis of longitudinal data in factorial experiments. Journal of Statistical Software, 50(12), 1-23. doi: 10.18637/jss.v050.i12

Priel, B., \& Shahar, G. (2000). Dependency, self-criticism, social context, and distress: Comparing moderating and mediating models. Personality and Individual Differences, 28, 515-525.

R Core Team. (2013). R: A language and environment for statistical computing. R Foundation for Statistical Computing, Vienna, Austria. http://www.Rproject.org/.

Robins, C. J., Keng, S. L., Ekblad, A. G., \& Brantley, J. G. (2012). Effects of mindfulness-based stress reduction on emotional experience and expression: A 
randomized controlled trial. Journal of Clinical Psychology, 68, 117-131. doi:10.1002/jclp.20857

Shapiro, S. L., Astin, J. A., Bishop, S. R., \& Cordova, M. (2005). Mindfulness-based stress reduction for health care professionals: results from a randomized trial. International Journal of Stress Management, 12(2), 164. doi: 10.1037/1072-5245.12.2.164

Shapiro, S. L., Brown, K., \& Biegel, G. (2007). Teaching self-care to caregivers: Effects of mindfulnessbased stress reduction on the mental health of therapists in training. Training and Education in Professional Psychology, 1(2), 105-115. doi: 10.1037/ 1931-3918.1.2.105

Shapiro, S. L., Brown, K. W., Thoresen, C., \& Plante, T. G. (2011). The moderation of mindfulness-based stress reduction effects by trait mindfulness: Results from a randomized controlled trial. Journal of Clinical Psychology, 67, 267-277. doi:10.1002/jclp.20761

Speca, M., Carlson, L. E., Goodey, E., \& Angen, M. (2000). A randomized, wait-list controlled clinical trial: The effect of a mindfulness-based stress reduction program on mood and symptoms of stress in cancer outpatients. Psychosomatic Medicine, 62, 613-622. doi: 0033-3174/00/6205-0613

Spijkerman, M. P. J., Pots, W. T. M., \& Bohlmeijer, E. T. (2016). Effectiveness of online mindfulness-based interventions in improving mental health: A review and meta-analysis of randomised controlled trials. Clinical Psychology Review, 45, 102-114. doi: 10.1016/j.cpr.2016.03.009
Teasdale, J. D., Segal, Z. V., \& Williams, M. G. (1995). How does cognitive therapy prevent depressive relapse and why should attentional control (mindfulness training) help? Behaviour Research and Therapy, 33, 25-39. doi:10.1016/0005-7967(94) E0011-7

Wahbeh, H., Svalina, M., \& Oken, B. S. (2014). Group, One-on-one or Internet?: Preferences for mindfulness meditation delivery format and their predictors. Open Medicine Journal, 1, 66-74. doi:10.2174/ 1874220301401010066

Wolever, R. Q., Bobinet, K. J., McCabe, K., Mackenzie, E. R., Fekete, E., Kusnick, C. A., \& Baime, M. (2012). Effective and viable mind-body stress reduction in the workplace: A randomized controlled trial. Journal of Occupational Health Psychology, 17, 246-258. doi:10.1037/a0027278

Zernicke, K. A., Campbell, T. S., Speca, M., McCabeRuff, K., Flowers, S., \& Carlson, L. E. (2014). A randomized wait-list controlled trial of feasibility and efficacy of an online mindfulness-based cancer recovery program: The eTherapy for cancer applying mindfulness trial. Psychosomatic Medicine, 76 , 257-267. doi:10.1097/PSY.0000000000000053

Zessin, U., Dickhauser, O., Garbade, S. (2015). The relationship between self-compassion and well-being: A meta-analysis. Applied Psychology: Health and Well-Being, 7(3), 340-364. doi:10.1111/ aphw.12051 